\title{
Customary physical activity and survival in later life: a study in Nottingham, UK
}

Centre for Ageing and Rehabilitation Studies, School of Health and Related Research, University of Sheffield, Community Sciences Centre, Northern General Hospital, Sheffield S5 7AU K Morgan

Leicestershire Mental Health Service NHS Trust, Department of Psychiatry, Leicester General Hospital, Leicester D Clarke

Correspondence to: Dr Kevin Morgan

Accepted for publication April 1997

\begin{abstract}
Objective-To assess the value of broadly based customary physical activity scores, derived from a questionnaire inventory, in predicting 10 year mortality among elderly people.

Design-A 10 year survival analysis of participants in the first wave of the Nottingham longitudinal study of activity and ageing who, in face to face interviews in 1985, provided detailed information on customary physical activity, health, and lifestyle.

Setting-Urban and suburban Nottingham Participants-A total of 1042 people aged 65 years and over randomly sampled from general practitioner records.

Main results-On the basis of factor scores derived from the interview questionnaire, activity levels were graded as "high", "intermediate", or "low". In Cox regression models controlling for age, health status, and cigarette smoking at the time of the activity assessment, these gradings were significantly related to 10 year survival. Relative to the "high" activity groups, the risk of dying was significantly increased in both the "intermediate" (hazard ratio (HR) 1.53 ; 95\% CI 1.12, 2.09) and "low" (HR 2.07; 95\% CI 1.53, 2.79) groups for women, and in the "low" group (HR 1.59; 95\% CI 1.12, 2.25) for men $(p<0.01$ throughout).
\end{abstract}

Conclusion-Since the survival model controlled for age, health status, and cigarette smoking, it is unlikely that the activity gradings used here are simple proxies for physical health. It is concluded, therefore, that within the elderly population, recall based survey assessments covering a wide range of customary or habitual physical activities, can provide indices showing both cross sectional utility and predictive validity.

(F Epidemiol Community Health 1997;51:490-493)

The physiological benefits of regular exercise and aerobic training have been demonstrated across the human lifespan. ${ }^{1}$ However, since the participation rates of elderly people in sports and formal exercise programmes are relatively low, ${ }^{2}$ the contribution to health and fitness of habitual or customary physical activity levels in later life merits particular attention, and has provided the focus for several recent cross sectional surveys. ${ }^{3-5}$ Typically, such studies employ recall based questionnaire assessments of physical activity. While this type of assessment appears to offer a practical research tool to explore relationships between physical fitness, health, and health gain among elderly people, data on the predictive value of such measurements remain scarce. ${ }^{6}$ In addition, the interpretation of positive cross sectional relationships between activity levels and health in later life remains difficult since, at the point of measurement, activity level may present as a proxy for, and thus confound with, physical health status. To address these issues, we examined relationships between activity gradings derived from a recall based questionnaire assessment of customary physical activity and 10 year survival in a stratified random sample of elderly people.

\section{Methods}

SAMPLE

Data were derived from the Nottingham longitudinal study of activity and ageing (NLSAA), the original sample for which was constructed as follows. Using electoral ward level statistics from the 1981 census, three areas of greater Nottingham were combined to provide a study population whose demographic composition (as regards age, sex, social class, ethnicity, and proportion of elderly people living alone) reflected the average national pattern for England and Wales. The resulting area included a total of 48733 individuals served by 25 general practitioners. With the consent and cooperation of these general practitioners, Nottinghamshire Family Practitioner Committee agesex lists were used to identify all noninstitutionalised individuals aged 65 years and over living within the survey areas. Of 8409 elderly people identified, 1299 eligible individuals (those alive and still living at the address provided) were randomly selected for interview. At sampling, those aged 75 years or older were intentionally over-represented in order to admit sufficient numbers for subsequent longitudinal analyses. Accordingly, while the ratio of "old" (65-74 years) to "very old" ( $75+$ years) people in the 1985 British population was approximately 1.62:1 (Office of Population Censuses and Surveys, $1983^{7}$ ), a baseline ratio of $1: 1$ was the target for this study.

The first (baseline) survey was conducted between May and September 1985. Of the 1299 individuals approached, 1042 were interviewed (a response rate of $80 \%$ ). Information on mortality within the baseline sample was provided by the National Health Service Central Register (NHSCR), which supplied copies of all death certificates as they accrued. 
Table 1 Mean values (minimum-maximum) by activity groupings for variables contributing to the CPA1 factor

\begin{tabular}{lccl}
\hline & \multicolumn{3}{l}{ Activity grouping } \\
\cline { 2 - 4 } Activity (units) & Low & Intermediate & High \\
\hline Women & $(\mathrm{n}=212)$ & $(\mathrm{n}=211)$ & $(\mathrm{n}=212)$ \\
Shopping (min/typical d) & $16.01(0-240)$ & $23.45(0-180)$ & $19.34(0-310)$ \\
Walking (min/typical d) & $26.65(0-248)$ & $43.54(0-240)$ & $46.93(0-385)$ \\
Flexibility (frequency: low-high) & $6.01(0-13)$ & $9.47(3-16)$ & $12.26(5-19)$ \\
Strength (frequency: low-high) & $4.37(0-12)$ & $9.36(2-16)$ & $11.73(4-18)$ \\
Indoor (min/wk) & $265.54(0-1260)$ & $519.41(0-1320)$ & $828.68(85-2100)$ \\
Outdoor (min/wk) & $8.25(0-240)$ & $53.03(0-840)$ & $225.67(0-1680)$ \\
Leisure (min/wk) & $22.83(0-210)$ & $59.84(0-360)$ & $154.25(0-1180)$ \\
& & & \\
Men & $(\mathrm{n}=135)$ & $(\mathrm{n}=136)$ & $(\mathrm{n}=135)$ \\
Shopping (min/typical d) & $17.09(0-240)$ & $19.41(0-180)$ & $17.11(0-230)$ \\
Walking (min/typical d) & $38.89(0-265)$ & $54.75(0-260)$ & $49.21(0-276)$ \\
Flexibility (frequency: low-high) & $6.4(0-13)$ & $10.14(5-16)$ & $12.88(7-18)$ \\
Strength (frequency: low-high) & $5.87(0-12)$ & $9.94(3-16)$ & $12.55(5-19)$ \\
Indoor (min/wk) & $150.73(0-1260)$ & $232.26(0-1500)$ & $428.48(0-3240)$ \\
Outdoor (min/wk) & $47.09(0-720)$ & $247.9(0-1440)$ & $785.79(0-2850)$ \\
Leisure (min/wk) & $38.83(0-1260)$ & $118.64(0-1365)$ & $226.48(0-1740)$ \\
\hline
\end{tabular}

QUESTIONNAIRE ASSESSMENT

Levels of customary physical activity (CPA) likely to promote muscle strength, joint flexibility, or stamina were assessed using detailed inventories. ${ }^{8}$ Continuous activities (those with a probable minimum energy cost of $2 \mathrm{kcal} / \mathrm{min}$, performed continuously for a minimum of three minutes, at least weekly, for at least the previous six weeks) were divided into five mutually exclusive functional categories: outdoor productive activities (eg gardening, house and car maintenance); indoor productive activities (eg housework, decorating, indoor maintenance); walking (purposeful walking outside the house or garden); shopping (ie continuous ambulatory behaviour associated with shopping); and leisure activities (eg cycling, swimming). In the assessment of walking, the interviewer asked in detail about walking done on the day prior to interview. If, however, this day had been atypical, then another was selected (up to a maximum of six days previously). At analysis, walking per se, and walking as shopping were divided into two separate categories. Both were scored as minutes per typical day.

In administering the questionnaire on outdoor, indoor, and leisure activities the interviewer first determined whether the respondent's participation in the activity met the criteria for "customary", and then asked in detail about the frequency and duration of participation. Each reported activity was scored as minutes per week. Non-participation was scored as zero. In addition, non-continuous activities likely to contribute to muscle strength (eg climbing high steps, dragging heavy loads) and joint flexibility (eg reaching for high shelves, bending for low shelves) were also included. Typically, these tasks form discrete units of physical activity and were therefore scored in terms of frequency of performance on a five point scale (ie performed never, occasionally, once or several times a week, daily, or several time a day).

General health was assessed using a 14 item health index scored from zero (no health problems) to 14 (multiple health problems) covering the presence or absence of: heart, stomach,
KEY POINTS

- Recall based assessments of habitual physical activity are increasingly being used in the study of wellbeing in later life.

- Data on the predictive validity of such assessments remain scarce.

- The present analyses demonstrate a clear relationship between questionnaire assessed activity level and 10 year mortality among elderly people.

- This relationship, independent of health status, provides evidence for the research utility of survey assessments of habitual activity.

eyesight, sleep, or foot problems; giddiness, headaches, urinary incontinence, arthritis and falls; long term disabilities and drug and walking aid use; and contact with (primary and secondary care) medical services. ${ }^{9}$ Smoking history (smoker/non-smoker) was assessed in a separate item. Interviews were followed by anthropometric measurements of handgrip strength and shoulder flexibility. As preliminary analyses had shown significant gender differences in activity patterns, data for men and women were analysed separately. All analyses were performed in SPSS version 6.0 for Windows. ${ }^{10}$

\section{SURVIVAL ANALYSES}

Factor analysis (principal components with varimax rotation) of the 1985 activity inventories extracted first principal components (labelled CPA1) which accounted for $33 \%$ of the variance in men, and $39 \%$ in women. ${ }^{4}$ Mean values (together with maximum/minimum scores) of those activities contributing to the factor CPA1 are shown in table 1 . For both men and women factor scores derived from CPAl showed significant $(r \geqslant 0.4 ; p<0.001)$ product moment correlations with instrumental measurements of handgrip strength and shoulder flexibility. ${ }^{4}$

In the 10 year period September 1985 to August 1995, the project received notification of 568 deaths ( 247 men and 321 women). To investigate relationships between activity and mortality, respondents were divided into three activity groupings according to tertile ranges for the $1985 \mathrm{CPA} 1$ factor scores. Mortality was then assessed in Cox regression models with survival (period from baseline assessment to death or censorship in August 1995) as dependent, and activity tertile groupings (high, intermediate, low), age (in 1985), health index scores, and smoking status (smoker/nonsmoker in 1985) as covariates. A forward stepwise approach to model selection was used, with entry into the model at the $5 \%$ level, and removal at the $10 \%$ level.

\section{Results}

For both sexes survival was significantly related to activity levels after controlling for age, health index score and smoking status (figure 1). Relative to the "high" activity groups, the risk 


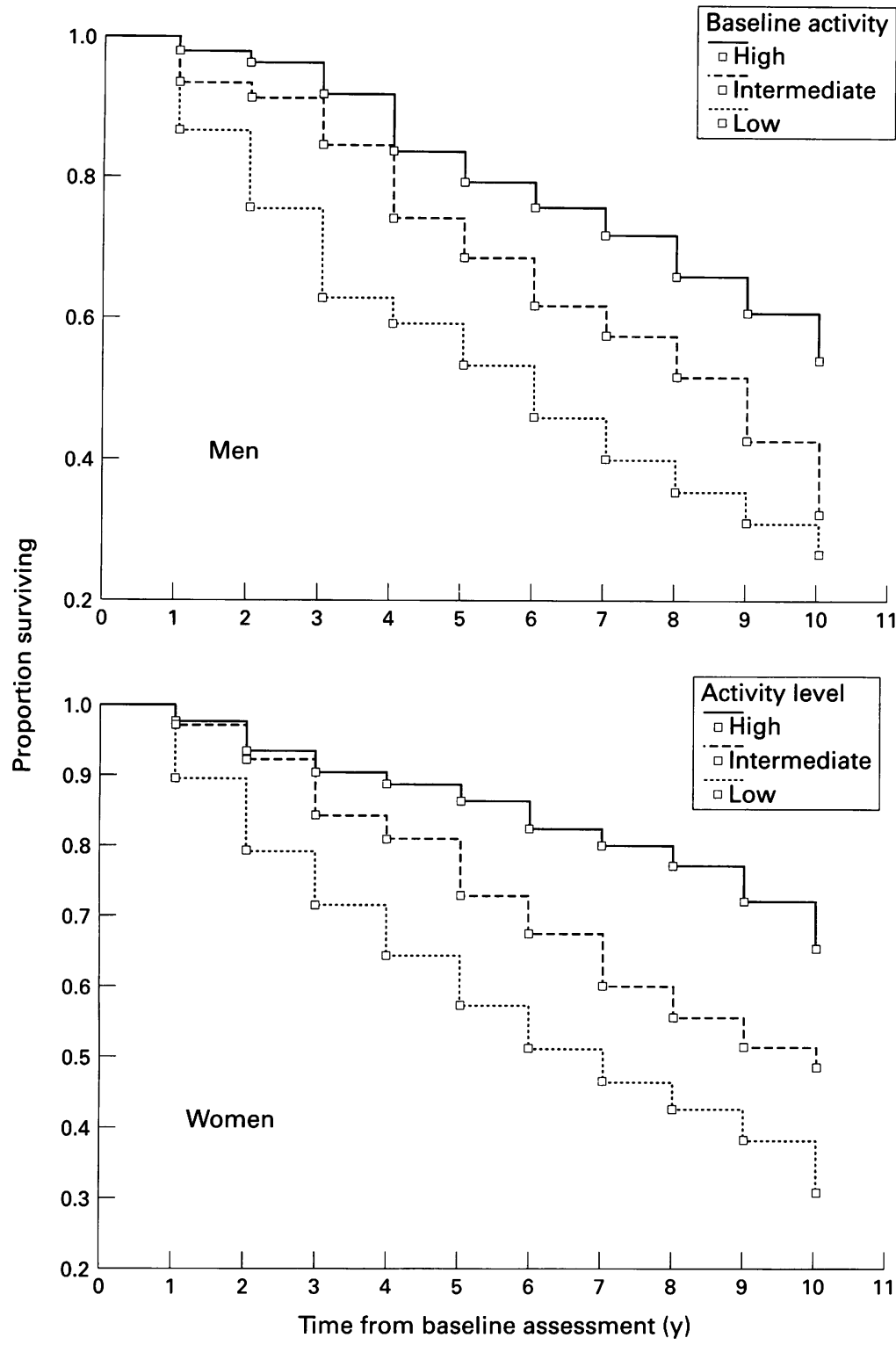

Figure 1 Survival functions (1985-95) for women and men grouped according to questionnaire assessed activity levels at baseline, controlling for age, health status, and cigarette smoking. justed estimates for low activity showed HR $2.75 ; 95 \%$ CI 1.98, $3.81 \quad(\mathrm{p}<0.0001)$ and for intermediate activity HR $1.73 ; 95 \%$ CI 1.23 , $2.43(\mathrm{p}=0.002)$.

\section{Discussion}

In recent years recall based questionnaire assessments (from which levels of fitness may be inferred) have increasingly been used to explore the role of physical activity in promoting health and psychological wellbeing in later life. While such assessments introduce the overlapping methodological issues of recall accuracy and criterion validity, prospective analyses of recall questionnaire data collected within this age group remain rare. The present results, therefore, provide useful evidence for the predictive validity of activity scores extracted from a broadly based survey assessment of older people. Since the survival model controlled for age, health status, and cigarette smoking at the time of the activity assessment, it is unlikely that the activity gradings used here are simple proxies for physical health. It remains possible, nevertheless, that the health index scores included in the analyses may have been insensitive to subclinical conditions or symptoms which, at the point of assessment, influenced activity level and ultimately, survival.

Unlike earlier work which has tended to focus only on a single source of exercise (typically, walking ${ }^{6}$ ), the present analyses used an index derived from a range of activities, each assessed for its physiological relevance. Such scores, we believe, reflect more accurately aspects of general activity. While the derivation of these scores does not allow for the contribution of any single source of activity (eg leisure, walking, housework, etc) to be identified as more or less important for longevity, it is clear from the table that, of those activities expressed in time units, indoor activities $(95 \%$ of which comprised housework ${ }^{8}$ ) and walking receive the greatest time commitment from individuals in this age group. Neither is it possible to assess, on the basis of the present all cause mortality data, relationships between CPA1 factor scores and specific causes of death. Recent evidence linking higher levels of walking with reduced cardiovascular mortality in old age ${ }^{11}$ indicates extremely complex pathways between activity and mortality requiring, at analysis, levels of statistical control beyond the scope of this study. With regard to the methodological objectives of the present analyses, however, we conclude that recall based survey assessments of customary or habitual physical activity can provide physiologically relevant indices of general activity level which, in elderly people, possess both cross sectional utility and predictive validity.

In a similar model unadjusted for age, health, or cigarette smoking, mortality was significantly increased in the "low" and "intermediate" activity groups for both sexes. Among women, unadjusted estimates for low activity showed HR 2.74; 95\% CI 2.07, $3.66(\mathrm{p}<0.0001)$ and for intermediate activity $\mathrm{HR} 1.70 ; 95 \% \mathrm{CI}$ $1.26,2.29$ ( $\mathrm{p}=0.0006)$. Among men, unad-

Funding: The Grand Charity; Help the Aged; PPP Charitable Trust; Trent Regional Health Authority. Conflicts of interest: none

1 Fentem PH, Turnbull NB, Bassey EJ. Benefits of exercise: the evidence. Manchester: University of Manchester Press, 1990. 
2 Allied Dunbar national fitness survey: main findings. London: Allied Dunbar (in association with the Health Education Authority and the Sports Council), 1992.

3 Baltes MM, Wahl, H-W, Schmid-Furtoss, U. The daily life of elderly Germans: activity patterns, personal control, and of elderly Germans: activity patterns, person

4 Morgan K, Dallosso H, Bassey EJ, Ebrahim S, Fentem PH, and Arie THD. Customary physical activity, psychological wellbeing, and successful ageing. Ageing Society 1991; 11 $399-415$

5 Ruuskanen JM, Ruoppila I. Physical activity and psychological well-being among people aged 65 to 84 years. $A g e$ Ageing 1995; 24:292-6.

6 Smith GD, Morris JN. Assessment of physical activity, and physical fitness, in population surveys. $\mathcal{F}$ Epidemiol Comm ${ }_{-}$ nity Health 1992; 26: 89-91.
7 Office of Population Censuses and Surveys. Census 1981: sex, age and marital status, Great Britain. London, HMSO, 1983.

8 Dallosso HM, Morgan K, Bassey EJ, Ebrahim SBJ, Fentem $\mathrm{PH}$, Arie THD. Levels of customary physical activity among the old and the very old living at home. $\mathcal{f}$ Epidemiol among the old and the very old living
Community Health 1988; 42: $121-7$.

9 Community Health 1988; 42: 121-7. ity of the shoulder joint measured as a range of abduction ity of the shoulder joint measured as a range of abduction years of age. Eur 7 Appl Physiol 1989; 58: 353-60.

10 Norusis MJ. SPSS for Windows: Professional Statistics, Release 6.0. Chicago: SPSS Inc, 1993.

11 LaCroix AZ, Leveille SG, Hecht JA, Grothaus LC, Wagner $\mathrm{EH}$. Does walking decrease the risk of cardiovascular disease hospitalizations and death in older adults? $\mathcal{F} A G S$ 1996; 44: 113-20. 